findings along with the literature would assist in making recommendations for this Paediatric app.

Results 27 papers were included in this review. Three papers were identified that studied apps specifically in aspects of Paediatrics. Overall, the studies showed that medical apps were an effective education tool by medical students during clinical placements. Survey results showed that medical apps were used by $43 \%$ of participants and $97 \%$ would like a Paediatric medical app to assist their learning during a Paediatric placement.

Conclusions Further long term studies are required to see the effects of medical apps in learning. More studies are also required to show how medical apps are beneficial for learning specifically during a Paediatric placement. A future Paediatric app should include concise information regarding common Paediatric conditions and have a simple design for quick use in practice.

\section{EARLY ONSET NEONATAL SEPSIS: EVALUATION OF THE KAISER PERMANENTE SEPSIS CALCULATOR FOR USE AT A TERTIARY NEONATAL UNIT IN THE UK}

Daniel Keen, Lucksini Selvadurai, Reham Hashem, Helen Gbinigie. UK

\subsection{6/bmjpo-2021-RCPCH.38}

Background The incidence of Early Onset Neonatal Sepsis (EONS) in the UK is currently estimated at $0.9 / 1000$ live births. NICE have published guidance (CG149) for treating infants based on antenatal risk factors and clinical indicators. In the UK, 13-20\% of infants are treated for suspected EONS based on CG149. This represents a significant burden, impacting on family bonding, breastfeeding, physical trauma through repeated cannulation, as well as on hospital bed status and finances. Covid-19 has compounded the issue, implementing restricted visitation disrupting the family unit. The Kaiser Permanente Sepsis Tool (KPT) was developed to determine the likelihood of infection based on multivariate analysis, has been used in the USA to successfully and safely to reduce the numbers of babies being prophylactically treated for this rare condition. Following on for their success we have now evaluated KPT for use within a UK demographic at Medway Maritime Hospital.

Objectives

1. Can KPT reduce prophylactic IV antibiotic use in well babies, when compared to NICE guideline CG149

2. Can KPT achieve this safely within our local demographic

Methods Data was collected from 62 newborn infants treated at Medway Maritime Hospital, between November 2019 and January 2020. These patients received prophylactic IV antibiotics as per NICE CG149. Inclusion criteria; $\geq 34$ weeks gestation and infants who were clinically well enough to be managed on the postnatal ward. The following data was obtained at the point of starting treatment; gestation, clinical examination, maternal peripartum temperature, rupture of membranes, maternal group B streptococcus. Subsequently peak CRP, blood and/or CSF culture results and duration of stay were recorded. Infants were classified into a risk category based on their peak CRP (Low <5, Medium 5-10, High $>10$ ). These findings were then compared to treatment recommended by the KPT (local incidence of EONS 1/1000 live births).
Results 16 infants were classed as high risk (26\%), 16 infants medium risk (27\%) and 29 infants low risk (47\%). The most common indication to treat was maternal pyrexia. Of infants screened, KPT recommended antibiotics for 2 infants (3\%); both of these were low risk. $47 \%$ of infants would have been kept under enhanced observations, with antibiotics being started if clinical symptoms developed. All blood and CSF cultures were negative. Of the infants classified as high risk, $50 \%$ of these infants would have been allocated to routine care. In the infants classified as high risk, there were no differences in the risk factors for neonatal sepsis, when compared with other risk categories.

Conclusions KPT represents a lucrative opportunity to reduce antibiotic use in well infants on the postnatal ward. However in line with similar studies, our results have highlighted that, just as with NICE CG149, is not infallible and liable to occasionally missing an asymptomatic child. Clinical vigilance is of the utmost importance and implementation of KPT would be have to marry with robust systems of neonatal observation and workforce training. A more conservative approach to EONS will invariably be associated with risk and ultimately it will be up to individual units to agree on what is acceptable.

\section{PATTERNS OF PRESENTATION OF SICKLE CELL DISEASE AT AHMED GASIM ESPECIAL IZED HOSPITAL FOR CHILDREN (2015-2016)}

Khalid AM Abdalbagi, Safaa Medani. Sudan

\subsection{6/bmjpo-2021-RCPCH.39}

Background Sickle cell disease is the commonest type of haemolytic anaemia in Sudan. It is commonly seen in those originating from western Sudan tribes, the affected children show various clinical presentations, laboratory findings, with varied complications and outcomes influenced by genetic, environmental and socioeconomic factors, which require a thorough assessment of patterns of presentations.

Objectives The aim of this study to determine the patterns of presentations of Sickle Cell Disease, in view of demographic data, clinical presentations, laboratory findings and the short outcome.

Methods This a prospective cross-sectional hospital based study, conducted in Ahmed Gasim Specialized Hospital for Children, (Khartoum North,Sudan) during the period from 1/ $11 / 2015$ to $31 / 1 / 2016$, covered all patients with Sickle cell disease, their ages were less than 18 years. Data collected by specifically designed questionnaires to fulfil the objectives of this study.

Results One hundred thirty eight patients were studied, aged between 9 months and 17 years with a mean age of 6.26 years ( \pm 4.44 SD). Nearly $93 \%$ of patients were belonging to western tribes of Sudan. The prevalence of sickle cell disease patients were $2.6 \%$ of total admission and $0.7 \%$ of the total number of patients seen in the Emergency Room in Ahmed Gasim Specialized Hospital for Children. Also anaemia due to Sickle Cell Disease comprises $43.9 \%$ of total anaemic patients. Seventy percent of patients were diagnosed during the first year of life. Painful crises were observed in 59\%, haemolytic crises seen in $20.4 \%$, sickle cell hepotpathy in $4.3 \%$, cerebrovascular accidents seen $1.4 \%$, sequestration crises in $0.7 \%$. Ninety fifth percent of patients presented with fever, $71 \%$ presented with fatigability, pain reported in $69.6 \%$, jaundice in 